\title{
First MDCT evidence of ruptured aberrant left subclavian artery aneurysm in right aortic arch, Kommerell's diverticulum and extrapleural hematoma treated by emergency thoracic endovascular aortic repair
}

\author{
Giacomo Sica $^{1}$, Giovanni Dialetto ${ }^{2}$, Giorgio Bocchini ${ }^{1}$, Giulia Lassandro ${ }^{1}$, Gaetano Rea ${ }^{1}$, \\ Massimo Muto ${ }^{1}$, Tullio Valente ${ }^{1}$ \\ ${ }^{1}$ Radiology Unit, Monaldi Hospital, Naples; ${ }^{2}$ Department of Cardiac Surgery, Monaldi Hospital, Naples, Italy
}

\begin{abstract}
Spontaneous ruptured aneurysm involving an aberrant subclavian artery with a right-sided aortic arch and Kommerell's diverticulum is a rare life-threatening condition that can be treated successfully if promptly identified. Multidetector Computed Tomography angiography is the first line imaging modality of thoracic vascular anomalies diagnosis. We report the case of a 74year-old man suffering from this emergency condition with medi-
\end{abstract}

Correspondence: Giacomo Sica, Radiology Unit, Azienda dei Colli,

Monaldi Hospital, 80131 Naples, Italy.

Tel. +39.081.7062829 - Fax: +39.081.7062857.

E-mail:gsica@sirm.org

Key words: Right-sided aortic arch; computed tomography angiography; endovascular repair.

Contributions: All the authors made a substantive intellectual contribution. All the authors have read and approved the final version of the manuscript and agreed to be accountable for all aspects of the work.

Conflict of interest: The Authors declare no conflict of interest or funding support.

Ethics approval and consent to participate: No ethical committee approval was required for this case report by the Department, because this article does not contain any studies with human participants or animals.

Informed consent: consent to CT exam and procedure was obtained from the patient included in this study.

Consent for publication: Not required as each image is anonymized and therefore the confidentiality of personal data is guaranteed.

Received for publication: 17 April 2020.

Accepted for publication: 8 July 2020.

${ }^{\circ}$ Copyright: the Author(s), 2020

Licensee PAGEPress, Italy

Monaldi Archives for Chest Disease 2020; 90:1322

doi: 10.4081/monaldi.2020.1322

This article is distributed under the terms of the Creative Commons Attribution Noncommercial License (by-nc 4.0) which permits any noncommercial use, distribution, and reproduction in any medium, provided the original author(s) and source are credited. astinal hematoma mostly extending to the left-side extrapleural cavity. The patient underwent successful emergency thoracic endovascular aortic repair and an Amplatzer vascular plug was placed into the first segment of the ALSA. Post-procedural imaging showed complete exclusion of the aneurysm. Emergency endovascular repair can be effective in such cases.

\section{Introduction}

Right aortic arch (RAA) is a rare anatomical variant occurring in 0.05 to $0.1 \%$ of radiology series $[1,2]$ and in $0.04 \%-0.1 \%$ of autopsy series [3]. About $50 \%$ of people with RAA also have an aberrant left subclavian artery (ALSA) originating from a Kommerell's diverticulum (KD) [4,5]; it is usually asymptomatic and incidentally detected. To the best of our knowledge, we describe the first thoracic endovascular aortic repair (TEVAR) in a patient with spontaneous ruptured ALSA aneurysm in RAA and $\mathrm{KD}$, complicated by a large extrapleural hematoma $(\mathrm{EPH})$.

\section{Case Report}

A 74-year-old man presented at emergency department with 6 hours of abrupt onset of acute chest pain, anemia, hypotension $(70 / 40 \mathrm{mmHg})$, and shortness of breath; the left hemithorax was dullness to percussion. Pulse was 160 beats/min, respiratory rate $36 / \mathrm{min}$; at blood tests $\mathrm{Ht}$ was $30.4 \%$ and $\mathrm{Hb} 6.6 \mathrm{~g} / \mathrm{dL}$. Cardiac enzymes and ECG findings were normal. The patient's condition rapidly deteriorated up to require intubation. After resuscitation procedures, an emergency multidetector computed tomography angiography (MDCTA) revealed a RAA (Figure 1) and a saccular ruptured aneurysm ( $7 \mathrm{~cm}$ maximum diameter) coming immediately off the origin of a retroesophageal ALSA arising from a KD (Figure 2). In the upper mediastinum there was an anomalous course of the left brachiocephalic vein passing posteriorly to the ascending aorta (AAo) and forming the superior vena cava with the right brachiocephalic vein (Figure 3). This complex anatomy was associated to a hematoma around the descending aorta (DAo) separating the parietal pleura from the endothoracic fascia, mostly extending to the left-side extrapleural space, forming a large $(10 \mathrm{x}$ $13 \times 32 \mathrm{~cm})$ EPH with typical "extrapleural fat" and "fat ghost ribs" signs, related to the inward displacement of the extrapleural fat stripe medially to the ribs by the EPH (Figure 4) [6,7]. Through the right femoral artery two endoprosthesis were deployed in a tel- 
escope modality with the proximal landing zone (LZ) just distal to the right subclavian artery and distal LZ in the proximal DAo (Ishimaru's Zone 4 or nobody's land), completely covering the aneurysm and the origin of the ALSA [8]. The subsequent angiography revealed no leak and initial sac thrombosis, confirmed by Transesophageal Echocardiography. To exclude retrograde patency of the aneurysm, an Amplatzer vascular plug was placed into the first segment of the ALSA (Figure 5). Twenty hours later a successful left carotid-axillary surgical bypass $(\mathrm{CAB})$ was performed $[9,10]$. After 15 days the patient was discharged without complications except for a brief transient tetraplegia completely recovered and is alive and healthy at 2-years follow-up.

\section{Discussion}

In the aortic arch (AA) anatomical variants the first diagnostic step is to evaluate the laterality of the arch, defined as the side of the trachea on which the arch crosses, and the main bronchi. Another rule is that the first arch branch vessel that contains a common carotid artery is contralateral to the AA, or that the retroesophageal or ALSA is always contralateral to the arch. RAA results from the persistence of the right fourth arch and right dorsal aorta and involution of the left fourth arch and dorsal aorta [1-3,11]. In an autopsy study, $50 \%$ of cases of RAA were associated with an ALSA, which can be retroesophageal (in $80 \%$ ), located between the trachea and the esophagus (in 15\%), or anterior to the trachea (in 5\%) [3]. Two anatomical RAA variants clinically differing in presentation, associations, and prognosis are described [11,12]:

Type 1 with "mirror-image branching", in which a left innominate artery arises as the first branch of the AA, and then divides into a left common carotid and left subclavian artery; in approximately $75 \%$ of these patients, cyanotic congenital anomalies (including tetralogy of Fallot, pulmonary stenosis with ventricular septal defects, tricuspid atresia, and truncus arteriosus) are present;

Type 2 with ALSA, more common, which arises as a fourth branch of the RAA or from an aortic diverticulum (KD); congenital heart anomalies are present in only $5 \%$ to $10 \%$.

In $10 \%$ of cases with a RAA and ALSA, there is a right ductus,

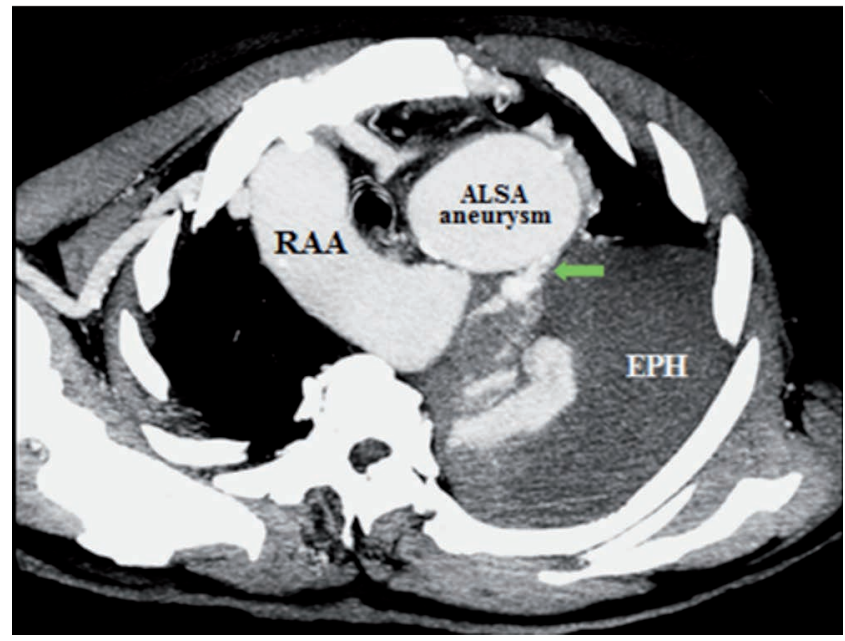

Figure 1. Maximum-intensity projection oblique axial reformat image shows a right aortic arch (RAA) and a ruptured (arrow) aneurysm of a retroesophageal aberrant left subclavian artery (ALSA). Discontinuity of ALSA aneurysm wall (green arrow) with extraluminal extravasation of contrast material and large haematoma in the posterior mediastinum, pleural and extrapleural spaces (EPH).
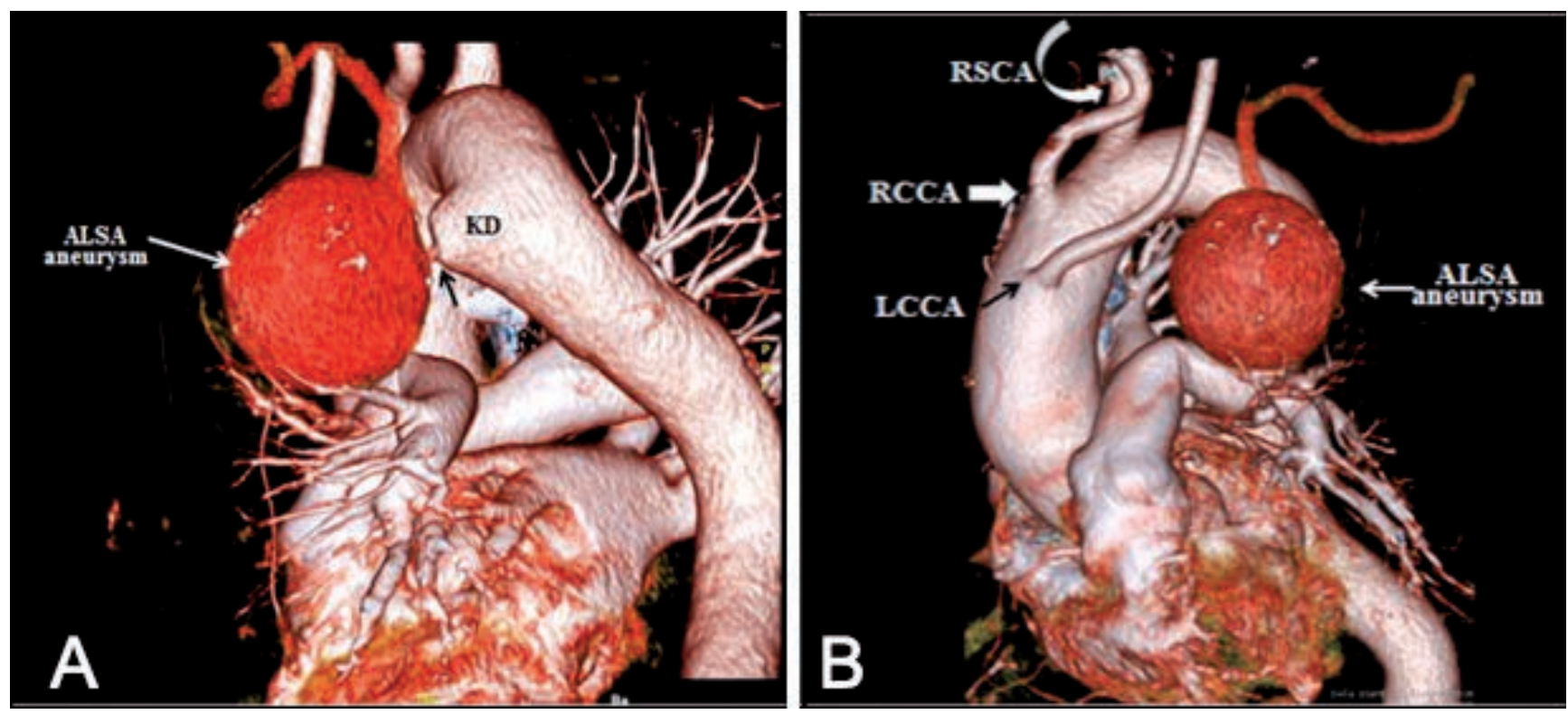

Figure 2. 3D volume rendering oblique sagittal reformat image. In (A) it is clearly appreciable how the aneurysm comes immediately off the aberrant left subclavian artery (ALSA) origin (arrow) from a Kommerell's diverticulum (KD); in (B) the right aortic arch branching pattern is the left common carotid (LCCA), right common carotid (RCCA) and right subclavian (RSCA) arteries followed by an ALSA aneurysm, which originates as the last branch from the proximal descending aorta and has a retroesophageal course to reach the left subclavian region. 


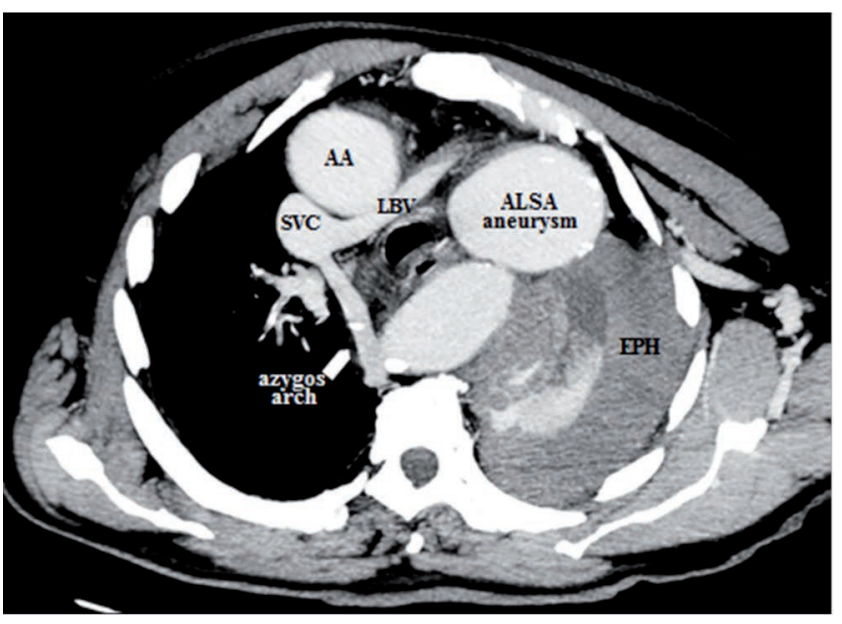

Figure 3. Maximum-intensity projection reformat axial image shows the anomalous course of the left brachiocefalic vein (LBV), posterior to the ascending aorta (AA) to form the superior vena cava (SVC). Hyperacute hypodense hematoma is associated to hyperdense extraluminal extravasation of contrast material.

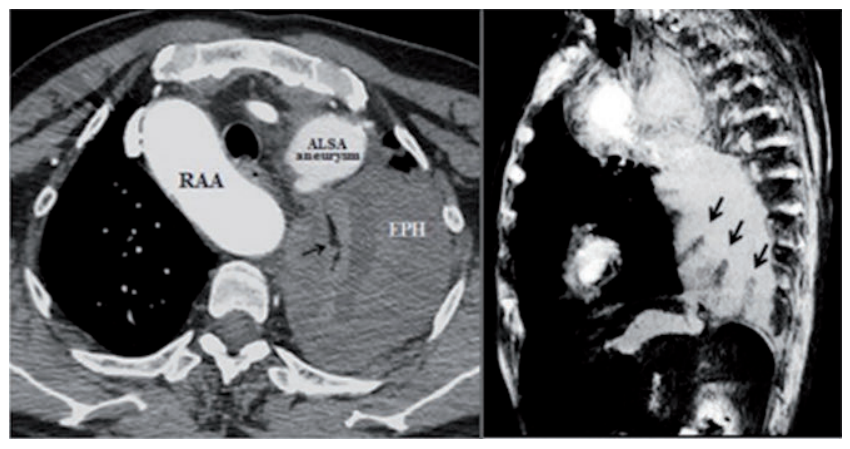

Figure 4. A) Maximum-intensity projection axial reformat image shows inward displacement of the extrapleural fat stripe (arrow) by extrapleural hematoma (EPH). B) Oblique sagittal minimumintensity projection reformat image shows "fat ghost ribs" sign (arrows) related to EPH; it is caused by a greater presence of displaced fat in the intercostal spaces compared to that present inside of the ribs.

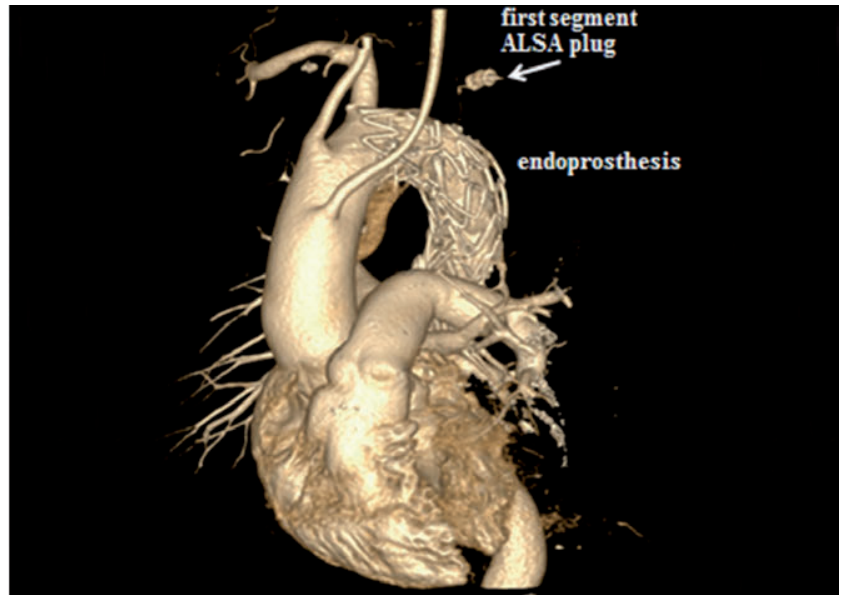

Figure 5. After thoracic endovascular repair (TEVAR) 3D volume rendering oblique sagittal reformat image shows complete aneurysmal exclusion and plug (arrow) in ALSA. without ring or associated intracardiac defect, but in $90 \%$ of the cases there is a left ductus, that is the second most common cause of a symptomatic vascular ring. In the adult population a RAA is often asymptomatic unless aneurysmal disease develops [13].

A meticulous analysis of vascular structure, obtained with a rigorous multiphasic MDCTA, is useful to define multiple combined vascular anomalies, their relationships with the trachea and esophagus, and it is essential for facilitate endovascular repair or surgical planning [14]. In our case the branches originate from the RAA in the following order: left and right common carotid arteries, right subclavian artery (RSCA) and ALSA which originates as the last branch from the proximal DAo (Type 2) and has a retroesophageal course to reach the left subclavian region $[1-3,12]$. The ALSA originates in the proximal DAo directly or more commonly from a bulbous diverticulum (KD), that represents the remnant of the left dorsal aortic root in the RAA and may to cause tracheal compression or dysphagia [15-17].

In adults, clinical implications of a RAA with ALSA and KD include esophageal compression, stridor, wheezing, dysphagia and, in the presence of atherosclerotic changes, complications such as dissection or aneurysmal dilatation up to vessels rupture [18]. Aberrant subclavian arteries are more prone to dilate than nonaberrant vessels and in the rupture of an aneurysmal aberrant subclavian artery a $100 \%$ mortality rate has been described [19]. One of the elective therapeutic options is its exclusion by TEVAR, preceded by a carotid-subclavian (or axillary) debranching bypass. In case of ruptured ALSA/diverticulum, the first-line treatment consists in TEVAR, followed by surgical bypass as soon as possible. The aim is to reduce the risk of spinal ischemia in cases of vertebral artery origin from the ALSA and to reduce the risk of upper left limb ischemia in cases of vertebral artery origin directly from the arch.

\section{Conclusions}

A correct contrast enhanced multiphasic MDCT is essential for the study of vascular anomalies allowing to obtain an adequate surgical planning of their complications even with less invasive and less time-consuming procedures such as TEVAR.

\section{References}

1. Knight L, Edwards JE. Right aortic arch: types and associated cardiac anomalies. Circulation 1974;50:1047-51.

2. Shuford WH, Sybers RG, Gordon IJ, et al. Circumflex retroesophageal right aortic arch simulating mediastinal tumor or dissecting aneurysm. Am J Roentgenol 1986;146:491-6.

3. Hastreiter AR, D'Cruz IA, Cantez T. Right-sided aorta. Part 1: Occurring of right aortic arch in various types of congenital heart disease. Br Heart J 1966;28:722-5.

4. Effmann EL, Whitman SA, Smith BR. Aortic arch development. RadioGraphics 1986;6:1065-89.

5. Kommerell B. [Verlagerung des Oesophagus durcheine abnorm verlaufende Arteria subclavia dextra (Arteria Lusoria)].[Article in German]. Fortschr Geb Roentgenstr 1936;54:590-5

6. Hammerman AM, Susman N, Strzembosz A, et al. The extrapleural fat sign: CT characteristics. J Comput Assist Tomogr 1990;14:345-7. 
7. Valente T, Tortora G, Bocchini G, et al. MDCT and US of intrathoracic extrapleural space soft tissue-containing lesions: US extrapleural fat sign and MDCT fat ghost ribs sign. Radiol Med 2017;122:479-86.

8. Fillinger MF, Greenberg RK, McKinsey JF, et al. Reporting standards for thoracic endovascular aortic repair (TEVAR). J Vasc Surg 2010;52:1022-33.

9. Shumacker HB Jr. Carotid axillary bypass grafts for occlusion of the proximal portion of the subclavian artery. Surg Gynecol Obstet 1973;136:447-8.

10. Criado FJ, Queral LA. Carotid-axillary artery bypass: a tenyear experience. J Vasc Surg 1995; 22:717-22.

11. Felson B, Palayew MJ. The two types of right aortic arch. Radiology 1963;81:745-59.

12. Bedford DE, Parkinson J. Right-sided aortic arch. Brit J Radiol 1936;9:776.

13. Cinà CS, Althani $\mathrm{H}$, Pasenau $\mathrm{J}$, Abouzahr L. Kommerell's diverticulum and right-sided aortic arch: a cohort study and review of the literature. J Vasc Surg 2004;39:131-9.
14. Carbone I, Sedati P, Galea N, et al. Right-sided aortic arch with Kommerell's diverticulum: 64-DCTA with 3D reconstructions. Thorax 2008;63:662.

15. Fisher RG, Whigham CJ, Trinh, C. Diverticula of Kommerell and aberrant subclavian arteries complicated by aneurysms. Cardiovasc Intervent Radiol 2005;28:553-60.

16. Tanaka A, Milner R, Ota T. Kommerell's diverticulum in the current era: a comprehensive review. Gen Thorac Cardiovasc Surg 2015;63:245-59.

17. Rea G, Valente T, Iaselli F, et al. Multidetector computed tomography in the evaluation of variants and anomalies of aortic arch and its branching pattern. Ital J Anat Embryol 2014;119:180-92.

18. Dikman SH, Baron M, Gordon AJ. Right aortic arch with ruptured aneurysm of anomalous left subclavian artery. J Cardiol 1974;34:245-9.

19. Myers JL, Gomes MN. Management of aberrant subclavian artery aneurysms. J Cardiovasc Surg 2000;41:607-12. 